\title{
Determine The Factors That Influence the Company's Failure by Using Logistic Regression
}

\author{
Ahmed Obaid Mahmood*,1, Obaid Mahmmood Alzawbaee ${ }^{2}$ \\ ${ }^{1}$ Department of Banking and Finance, Al-Maarif University College, Iraq \\ ${ }^{2}$ University of Jihaan, Sulaimanya, Iraq \\ *ahmed.obed@uoa.edu.iq
}

\begin{abstract}
KEYWORDS: $\quad$ Logistic Regression, Successful Companies, Failed Companies, Prediction, Ranking.
\end{abstract}
\begin{abstract}
:
Predicting the failure of companies is one of the important and influencing matters, whether for the companies themselves, competing companies or investors. In addition, to the effect of this on the country's economies. In our research, the logistic regression technique was applied to know the most important variables that have a significant effect on the failure or success of companies. The research also includes the arrangement of these variables according to their importance in influencing the dependent variable that represents the failure or success of companies. The application was applied to a sample with size of (33) companies that included (16) successful companies and (17) failed companies. However, four quantitative variables were specified as well as the dependent variable (Y), which is a qualitative variable. And that the model which was built proved the tests significantly and its ability to classify correctly by $84.8 \%$.
\end{abstract}

\section{REFERENCES:}

[5] Ahan, A.E; \& Okafor, R. (2010): Application of Logistic Regression Model to Graduating (CGPA of University Graduate-University of Lagos). Journal of Modern Mathematics and Statistics, 2(2), pp. 58 - 62.

[6] Andrews, D. W.(1988b). 'Chi-Square Diagnostic Tests for Econometric Model Theory', Econometrica, Vol. 56, pp. 1419-53.

[7] Brown , C.E (1998): "Applied Multivariate, statistics in Geohydrology and related sciences ", Springer - verlag. Berlin Heidelberg, chapter 6, multiple regression. pp. 62-66.

[8] Dutta A., and Bandopadhyay G., (2012). Performance in the Indian Stock Market Using Logistic Regression", International Journal of Business and Information, Vol. 7, No. 1, June, 105-136.

[9] Fagoyinbo, I.S, Ajibode, I.A., Olaniran, Y.O.A , (2014). The Application of Logistic Regression Analysis to the Cummulative Grade Point Average of Graduating Students: A Case Study of Students' of Applied Science, Federal Polytechnic, Ilaro-Developing Country Studies, Vol.4, No.23, 26-30.

[10] Hosmer, D. W. and Lemeshow, S. Applied Logistic Regression, Wiley, N. Y, $1989 . \quad$ King, J.E (2003). "Running A Best-Subsets Logistic Regression: An Alternative to Stepwise Methods", Educational and Psychological Measurement, Vol. 63, No. 3, June, 392-403

[11] Hosmer, D. W. and Lemeshow, S. (1989) Applied Logistic Regression, Wiley, N. Y. King, J.E. (2002). " Logistic Regression: Going beyond point-and-click", Paper presented at the annual Meeting of the American Educational Research Association, New Orleans, LA.

[12] Li, H., Sun, J. and Wu, J. (2010). "Predicting business failure using classification and regression tree: An empirical comparison with popular classical statistical methods and top classification mining methods", Expert Systems with Applications, Vol. 37, No. 8, August, 5895- 5904.

[13] Litwin, H., \& Sapir, E. V. (2009). Perceived income adequacy among countries: Findings from the survey of health, ageing, and retirement in Europe. The Gerontologist, 49, 397-406. doi:10.1093/geront/gnp036. 
[14] Nummela, O. P., Sulander, T. T., Heinonen, H. S., \& Uutela, A. K. (2007). Self-rated health and indicators of SES among the ageing in three types of communities. Scandinavian Journal of Public Health, 35, 39-47. doi:10.1080/14034940600813206.

[15] Osbome W., Jason, (2012). Logits and tigers and bears, oh my! A brief look at the simple math logistic regression and how it can improve dissemination of results, Practical Assessment, Research \& Evaluation,17(11), 1-10.

[16] Stoller, M. A., \& Stoller, E. P. (2003). Perceived income adequacy among elderly retirees. Journal of Applied Gerontology, 22, 230-251. 


\title{
تحديد العو امل المؤثرة على فشل الشر كات باستخدام الإنحدار اللوجستي
}

\author{
م.م. احمد عبيد محمود،1، أ.د. عبيد محمود الزوبعي2

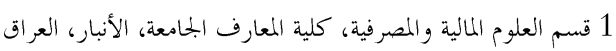 \\ 2 جامعة جيهان، السليمانية، العراق \\ *ahmed.obed@uoa.edu.iq
}

الكلمات المفتاحية | الإنحدار اللوجستي، الشركات الناجحة، الشركات الفاشلة، تنبؤ، تصنيف.

Crossref doi https://doi.org/10.51345/.v32i2.367.g228

\section{ملخص البحث:}

ان التنبؤ بفشل الشر كات يعد من الامور المهمة والمؤثرة سواء للشر كات نفسها او الشركات المنافسة او المستثمرين اضافة الماتل

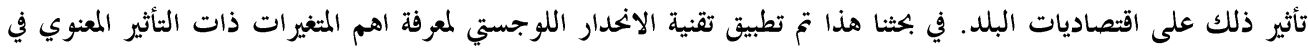

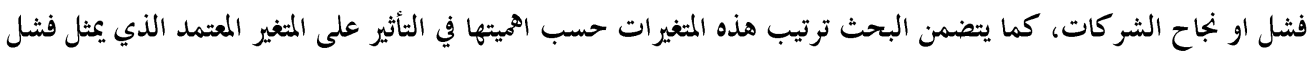

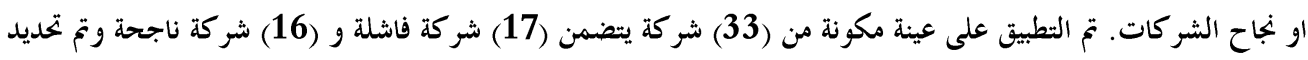

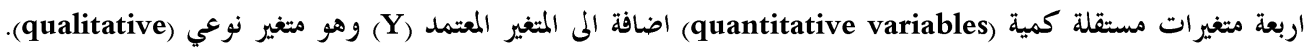

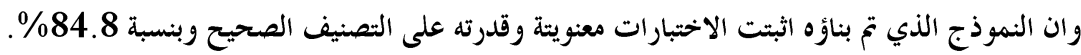

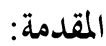

ان استخدام تحليل الانحدار هـدف بناء نموذج للتنبؤ بقيم المتغير المعتمد قد استخدم ويستخدم في العديد

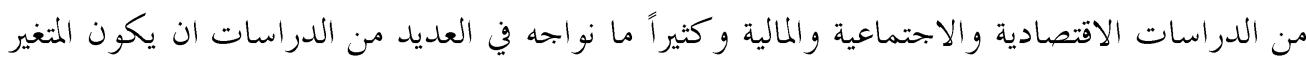

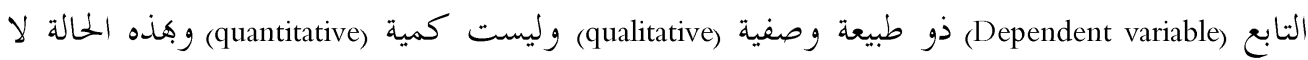
تتحقق فرضيات ومتطلبات نماذج الانحدار (regression model). وقد جاء استخدام الانحدار اللوجستي

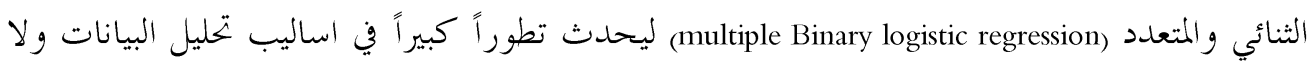

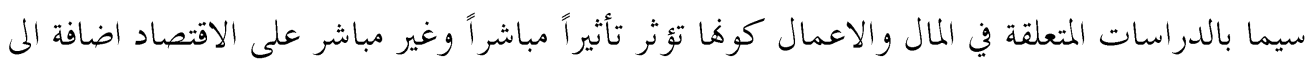
دورها في عمليات التنمية لما توفرة من فرص استثمارية وتمويلية. ان التنبؤ بفشل الشركات لهير له اهمية كبيرة

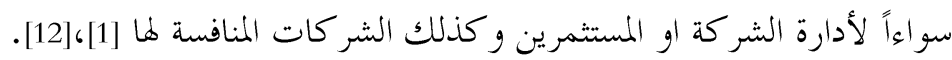
في بكثنا هذا يتم استخدام الانحدار اللوجستي لغرض بناء نموذج يمكن بواسطتة التنبؤ بفشل الشركات

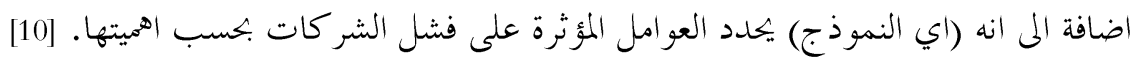


و تتحدد مشكلة البحث من خلال:

- المتغيرات ذات التأثير المعنوي على فشل الشركات

- ترتيب هذه المتغيرات حسب الاهمية في التأثير

و يعتمد البحث على الفرضية التالية:

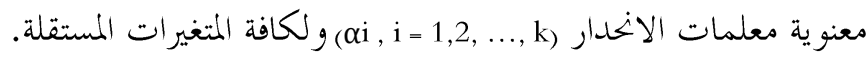

ويهاف البحث الى بناء نموذج احصائي يمكن بواسطتة التنبؤ بفشل الشركات و التعرف على المتغيرات ذات التأثير المعنوي وترتيبها حسب الاهمية بأستخدام تحليل الانحدار اللوجستي. البحث يعتبر اداة فاعلة لأتخاذ القرار المناسب بحاة الشركات سواءاً من قبل ادارقا او المستثمرين او الشركات المنافسة والمتمثلة بالبيع او التصفية او القرارات التصحيحية. و يعتمد البحث المنهج التحليلي الاستنتاجي من خلال تطبيق اسس النظرية الاحصائية المتمثلة بتحليل الانحدار اللو جسيتي.

الاطار النظري:

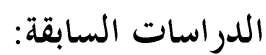

تباينت الدراسات التي تناولت الشركات المالية او شركات الاسهم في نوعية البيانات المستخدمة في التحليل وطبيعة الاداة التحليلية وفي ادناه نبذة من تلك الدراسات: اكدت الدراسة [15] 2012 على الاهمية المتزايدة لأعتماد النموذج اللوجستي في الدراسات الانسانية في الوقت الحاضر وتم استخدام مثال توضيحي على كيفية التعامل مع المخرجات التي يمكن الخصول عليها بأستخدام التحليل اللوجستي. اعتمدت الدراسة [2]،2014 على بناء نموذج الانحدار اللوجستي لبيان العوامل المؤثرة على اداء الاسهم في الاوراق المالية في سوق الكويت لغرض توفير اداة فاعلة للمستثمرين لأتخاذ القرارات الاستثمارية الملائمة لتملك الاسهم او بيعها بالاضافة الم اتخاذ الاجراءات التصحيحية حيال وصفها. استخدمت الدراسة [3] 2015 النموذج اللوجستي لتشخيص العوامل التي تحدد فترة بقاء المصابين .مرض السرطان في المستشفى واعتمدت الدراسة عينة بحجم (73) مصاباً .مرض السرطان في محافظة بغداد خلال الفترة (2011 - 2010) وتوصلت الدراسة الى ان المتغيرين المؤثرين معنوياً في اصابة المريض هما متغير العلاج و متغير فقر الدم. 
استخدمت الدراسة [8] 2012 الانحدار اللوجستي للتنبؤ بأداء الاسهم في سوق الاسهم الهندية حيث اعتمدت على بعض النسب المالية كمتغيرات مستقلة وشملت عينة الدراسة (30) شركة من الشركات ذات القيمة السوقية الكبيرة خلال فترة اربع سنوات حيث توصلت الدراسة الى ان استخدام الانحدار اللو جستي يساعد على تكوين رأي حول استثمار الاسهم.

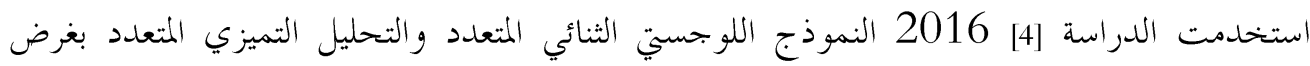
المفاضلة بينهما وتمثل بحتمع الدراسة بجميع الاسر بولاية الخرطوم وعينة الدراسة تكونت من (545) مفردة وتوصلت الدراسة الى ان نتائج النموذج اللوجستي افضل من الدالة التميزية في تحليل البيانات ذات المتغير التابع النوعية.

وتناولت الدراسة [1] 2019، بناء نموذج احصائي للتنبؤ بأداء الاسهم بالسوق الاردني والتعرف على لتئ العوامل ذات التأثير المعنوي حسب ترتيبها واهميتها وتوصلت الى ان نسبة الديون والقيمة الدفترية للاسهم هما المتغيران المعنو يان.

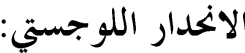

الانحدار اللوجستي يستخدم لفحص وبناء العلاقة بين المتغير التابع (Dependent variable) النوعي (quantitative or اياً كان نوعها كمية او نوعية (Independent variables) والمتغيرات (qualitative)

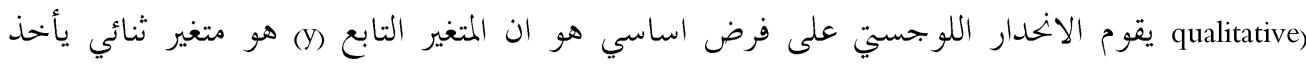

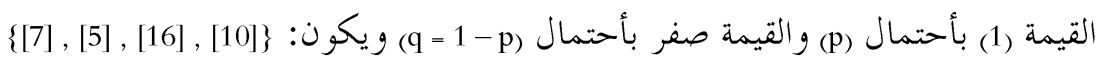

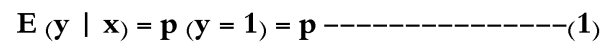

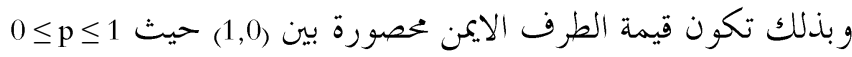

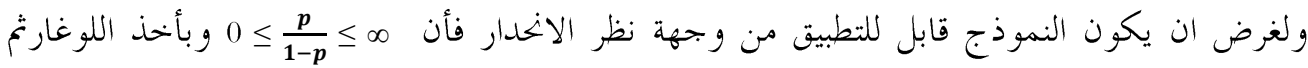

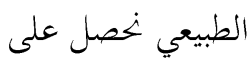

$$
---(2) \infty \leq \ln \left(\frac{p}{1-p}\right) \leq-\infty
$$

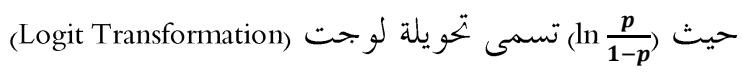
ويمكن كتابة نموذج الانحدار في حالة وجود عدد من لمن المتغيرات المستقلة كما يلي:

$$
\begin{array}{r}
\operatorname{Ln}\left(\frac{p}{1-p}\right)=B_{0}+\sum_{i=1}^{n} B j X_{i j}-- \\
J=1,2, \ldots ., \mathrm{k} \\
\mathrm{I}=1,2, \ldots \ldots \mathrm{n}
\end{array}
$$


ان الانحدار اللوجستي هو اداة قوية لانه يقدم اختباراً لمعنويات المعلمات كما انه يعطي الباحث فكرة عن مقدار تأثير المتغير المستقل على المتغير التابع بالاضافة المى انه يرتب تأثيرات المتغيرات المستقلة، وانه اي الانحدار اللوجستي اقل حساسية بتحاة الاخر افات عن التوزيع الطبيعي (Normal Distribution) و العلاقة الخطية لمتغيرات البحث. \}[9] , [15] , [11] يستخدم نموذج الانحدار اللوجستي في التنبؤ بأحتمال حدوث حدثرث معين بتوفيق البيانات بشكل منحني لوجستي اي ان اعتماد الانحدار على المنحنى اللوجستي الذي يأخذ الصيغة التالية: $P=\frac{e^{a+B x}}{1+e^{a+B x}}=\frac{1}{1+e^{a+B x}}$ بدلاً من معادلة الخط المستقيم (e) الجحانب التطبيقي:

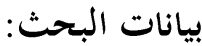

تمثلت بيانات البحث بيانات (33) شركة (17) منها شركات فاشلة و(16) اخرى لشر كات ناجحة وقد . تمثلت المتغيرات كما يأتي: (Dependent variable) المتغير المعتمد: Y 1 = Y 0=Y اما المتغيرات المستقلة فقد تم دراسة اربعة متغيرات مستقلة (Independent variables) و كما يأتي: X1

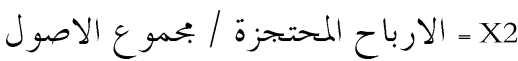
X3

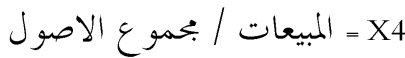
والجدول رقم (1) يمثل بيانات البحث 
الجحدول رقم (1) بيانات البحث X3 X3 X

\begin{tabular}{|c|c|c|c|c|}
\hline$Y$ & $\mathrm{X} 1$ & $\mathrm{X} 2$ & X3 & $\mathrm{X} 4$ \\
\hline 1 & 0.523 & 0.034 & 0.036 & 0.47 \\
\hline 1 & 0.539 & -0.07 & 0.024 & 0.52 \\
\hline 1 & 0.799 & -0.17 & -0.09 & 0.29 \\
\hline 1 & 0.626 & -0.27 & -0.04 & 0.65 \\
\hline 1 & 0.733 & -0.26 & -0.17 & 0.33 \\
\hline 1 & 0.72 & 0.095 & 0.047 & 0.42 \\
\hline 1 & 0.723 & 0.128 & 0.136 & 1.03 \\
\hline 1 & 0.321 & 0 & -0.268 & 0.367 \\
\hline 1 & 0.399 & 0.482 & 0.589 & 0.97 \\
\hline 1 & 0.257 & 0.133 & 0.163 & 2.547 \\
\hline 1 & 0.135 & 0.084 & 0.105 & 4.128 \\
\hline 1 & 0.486 & 0.101 & 0.122 & 5.116 \\
\hline 1 & 0.035 & 0.154 & 0.0003 & 0.026 \\
\hline 1 & 0.057 & -0.086 & 0.102 & 1.044 \\
\hline 1 & 0.033 & 0.151 & -0.001 & 0.0188 \\
\hline 1 & 0.122 & 0.81 & 0.255 & 0.432 \\
\hline 0 & -0.118 & -0.008 & -0.12 & 0.64 \\
\hline 0 & 0.053 & 0 & 0.006 & 0.078 \\
\hline 0 & -0.049 & 0 & -0.048 & 0.126 \\
\hline 0 & 0.176 & 0.032 & 0.011 & 0.035 \\
\hline 0 & 0.191 & 0.029 & 0.009 & 0.028 \\
\hline 0 & -0.068 & -0.265 & -0.038 & 1.217 \\
\hline 0 & -0.344 & -0.777 & -0.184 & 1.636 \\
\hline 0 & 0.011 & 0.223 & 0.046 & 0.607 \\
\hline 0 & -0.03 & -0.61 & -0.28 & 0.44 \\
\hline 0 & -0.13 & -0.11 & -0.11 & 0.34 \\
\hline 0 & -0.16 & -0.91 & -0.27 & 0.37 \\
\hline 0 & 0.272 & -0.17 & -0.08 & 0.28 \\
\hline 0 & 0.247 & -0.3 & -0.01 & 0.31 \\
\hline 0 & 0.322 & -0.1 & -0.1 & 0.19 \\
\hline 0 & 0.336 & -0.57 & -0.37 & 0.32 \\
\hline 0 & 0.665 & 0.061 & 0.112 & 0.27 \\
\hline 0 & 0.215 & 0.055 & 0.099 & 0.249 \\
\hline
\end{tabular}

\section{عرض وتفسير النتائج:}

بأستخدام البرنامج الجاهز SPSS لتحليل البيانات ولغرض تقدير معلمات النموذج تم اعتماد التدوير و اعتماد مشتقة دالة الامكان الاعظم لأقل قيمة سالب ضعف لوغاريثم دالة الامكان الاعظم (Iteration) 
(2log likelihood) حيث تم الحصول على التقدير الامثل لمعلمات النموذج وان: = 2log likelihood -) 21.743)

في الدورة السابعة التي توقفنا عندها لان التغير في المعلمات (B0 , B1 , B2 , B3 , B4) اصبح صفر، بأعتباره افضل نتيجة يمكن الحصول عليها للمعلمات وان (2log likelihood - هي في فايتها الصغرى و كما يوضح ذلك الجمدول رقم (2).

Iteration History (2) الجدول رقم

Coefficients

\begin{tabular}{llllllll} 
Iteration & & -2 Log likelihood & Constant & $\mathrm{x} 1$ & $\mathrm{x} 2$ & $\mathrm{x} 3$ & $\mathrm{x} 4$ \\
\hline Step 1 & 1 & 27.752 & -1.102 & .543 & 2.117 & -.518 & 3.064 \\
\cline { 2 - 7 } & 2 & 23.818 & -1.804 & 1.114 & 4.679 & -2.715 & 4.620 \\
\cline { 2 - 7 } & 3 & 22.359 & -2.355 & 1.940 & 6.896 & -4.765 & 5.465 \\
\cline { 2 - 7 } & 4 & 21.782 & -2.846 & 3.037 & 8.520 & -6.490 & 5.861 \\
\hline & 5 & 21.743 & -3.028 & 3.374 & 9.208 & -7.109 & 6.088 \\
\hline & 6 & 21.743 & -3.039 & 3.393 & 9.263 & -7.149 & 6.106 \\
\hline
\end{tabular}

a. Method: Enter

b. Constant is included in the model.

c. Initial -2 Log Likelihood: 45.717

d. Estimation terminated at iteration number 7 because parameter estimates changed by less than .001 .

والجحدول رقم (3) يوضح معلمات النموذج (العمود B)، والخطأ المعياري (S . E) لكل معلمة وأحصاءة و الد Wald Statistics) اضافتاً الى معنوية المعلمات (Sig) و القيمة الاسية للمعلمة (BXP(B) و التي سنفسرها لاحقاً.

Variables in the Equation (3) الجدول رقم

\begin{tabular}{ccc|c|c|c|c|c|c|c} 
& & & & & & \multicolumn{3}{|c}{$95 \%$ C.I.for EXP(B) } \\
\hline \multirow{2}{*}{$\begin{array}{c}\text { Step } \\
1 \mathrm{a}\end{array}$} & $\mathrm{x} 1$ & 3.394 & 2.031 & 2.792 & 1 & .095 & 29.772 & .556 & 1594.429 \\
\cline { 2 - 11 } & $\mathrm{x} 2$ & 9.264 & 5.042 & 3.376 & 1 & .066 & 10548.284 & .539 & 206569844.726 \\
\cline { 2 - 10 } & $\mathrm{x} 3$ & -7.149 & 6.805 & 1.104 & 1 & .293 & .001 & .000 & 487.312 \\
\hline & $\mathrm{x} 4$ & 6.106 & 2.261 & 7.291 & 1 & .007 & 448.682 & 5.333 & 37749.701 \\
\hline $\begin{array}{c}\text { Constan } \\
\mathrm{t}\end{array}$ & -3.039 & 1.270 & 5.722 & 1 & .017 & .048 & & \\
\hline
\end{tabular}

a. Variable(s) entered on step 1: x1, x2, x3, x4. 
ولاختبار كفاءة النموذج وجودتة (goodness of fit) حيث ان في حالة الانحدار اللوجستي يتم استخدام لوغاريثم نسبة الامكان الاعظم (log likehood ratio) الذي يتبع توزيع مربع كاي (Chi-Square 22) وفق العلاقة:

$$
\chi^{2}=2\left\{\log _{\mathrm{e}} \mathbf{1}_{0}-\log _{\mathrm{e}} 1_{1}\right\}
$$

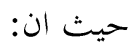

L1 : قيمة دالة الامكان الاعظم الذي يحتوي على (i) متغير L0 قيمة دالة الامكان الاعظم الذي يكتوي على (1-i ) متغير وبلغت قيمة (23.974=23) وهي معنوية كما يبين الجحدول رقم (4) الآتي:

Omnibus Tests of Model Coefficients (4) الجدول رقم

\begin{tabular}{ccc|c|c} 
& & Chi-square & df & Sig. \\
\hline \multirow{3}{*}{ Step 1 } & Step & 23.974 & 4 & .000 \\
\cline { 2 - 5 } & Block & 23.974 & 4 & .000 \\
\cline { 2 - 5 } & Model & 23.974 & 4 & .000 \\
\hline
\end{tabular}

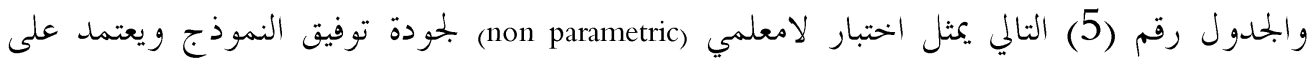

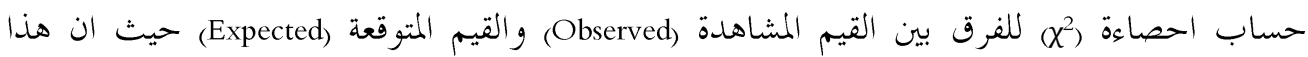

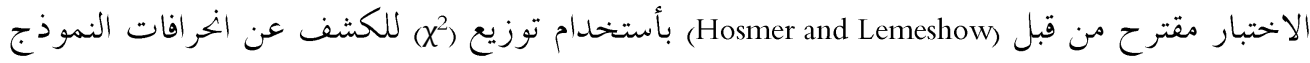

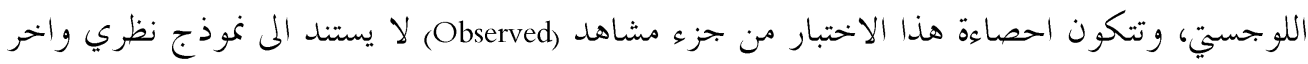
متوقع (Expected) محسوب من تقديرات النموذج اللوجستي وتحسب احصاءة (2) لجودة التوثيق.

Contingency Table for Hosmer and Lemeshow Test (5) الجدول رقم

$$
\mathrm{y}=0
$$

$$
\mathrm{y}=1
$$

\begin{tabular}{ccc|c|c|c|c} 
& & Observed & Expected & Observed & Expected & Total \\
\hline Step 1 & 1 & 3 & 2.991 & 0 & .009 & 3 \\
\cline { 2 - 7 } & 2 & 3 & 2.837 & 0 & .163 & 3 \\
\cline { 2 - 7 } & 3 & 3 & 2.686 & 0 & .314 & 3 \\
\hline 4 & 3 & 2.454 & 0 & .546 & 3 \\
\hline 5 & 1 & 2.331 & 2 & .669 & 3 \\
\hline & 2 & 2 & 2.008 & 1 & .992 & 3 \\
\hline
\end{tabular}




\begin{tabular}{c|c|c|c|c|c|c}
\hline 8 & 1 & .499 & 2 & 2.501 & 3 \\
\hline 9 & 0 & .292 & 3 & 2.708 & 3 \\
\hline 10 & 0 & .049 & 6 & 5.951 & 6 \\
\hline
\end{tabular}

من بحاميع المتغير التابع الثنائي (y) مع بحاميع الاحتمالات التقديرية. و من الجلدول رقم (6) نلاحظ ان

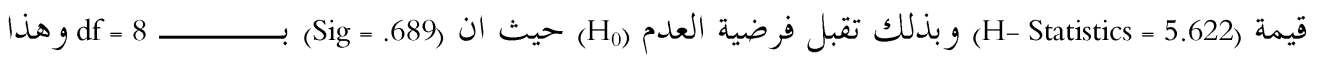
يؤ كد جودة التوفيق للنموذج بالكامل.

Hosmer and Lemeshow (6) الجدول رقم

\begin{tabular}{rrrr} 
Step & Chi-square & Df & Sig. \\
\hline 1 & 5.622 & 8 & .689 \\
\hline
\end{tabular}

ولمعرفة مدى امكانية النموذج الذي تم بناؤه على تصنيف المفردات فأن الجمدول رقم (7) التالي يوضح ذلك، حيث بلغت النسبة المئوية للتصنيف الصحيح (84.8\%) اي ان عدد المشاهدات المصنفة بشكل صحيح هي (28) مفردة بينما هناك (5) مفردات صنفت بشكل خاطئ.

Classification Table (7) الجدول رقم

\begin{tabular}{|c|c|c|c|c|c|}
\hline & & & \multicolumn{3}{|c|}{ Predicted } \\
\hline & \multirow{2}{*}{\multicolumn{2}{|c|}{ Observed }} & $\mathrm{y}$ & & Percentage \\
\hline & & & 0 & 1 & Correct \\
\hline \multirow[t]{3}{*}{ Step 1} & \multirow[t]{2}{*}{$\mathrm{y}$} & 0 & 15 & 2 & 88.2 \\
\hline & & 1 & 3 & 13 & 81.3 \\
\hline & $\mathrm{Ov}$ & entage & & & 84.8 \\
\hline
\end{tabular}

a. The cut value is .500

من الجحدول رقم (3) نلاحظ ان العمود (B) يحتوي على معلمات النموذج بوحدات (Log-odds) وان

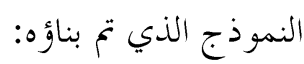

$$
\log \left(\frac{p}{1-p}\right)=-3.039 \text { + } 3.394 \text { x1 + } 9.264 \text { x2 - } 7.149 \text { x3 + } 6.106 \text { x4 -------------- (6) }
$$

وتشير النتائج الظاهرة في نفس الجمدول ان المتغيرات المستقلة التي ها تأثير معنوي على بلى بناح او فشل

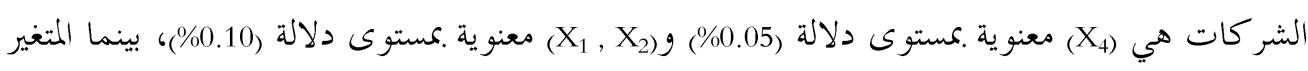




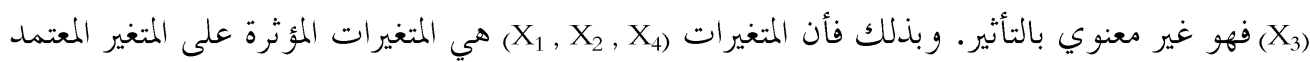

$$
\begin{aligned}
& \mathrm{B}_{\mathbf{0}}=-3.039-----\mathrm{EXP}=\mathbf{0 . 0 4 8} \\
& B_{4}=6.106-----E X P=488.682 \\
& B_{1}=3.394----E X P=29.772 \\
& B_{2}=9.264----E X P=10548.284
\end{aligned}
$$

وان قيمة المعلمات هي (Y)

- احتل المتغير (X) و الذي يمثل المبيعات / بحموع الاصول المرتبة الاولى في التأثير على المتغير (Y)

$$
\text { وان هذا المتغير له معلمة ذات تأثير معنوي عند (م) (م=0.05). }
$$

- جاء المتغير (X2) الذي يمثل الارباح المحتجزة / بحموع الاصول بالمرتبة الثانية بالتأثير على المتغير

$$
\text { التابع وان معلمة هذا المتغير موجبة ومعنوية عند (م=0.10) . }
$$

- المتغير (X) و الذي يمثل صافي رأس المال العامل / بحموع الاصول جاء بالمرتبة الثالثة بالتأثير على

$$
\text { المتغير التابع (Y) وان معلمة هذا المتغير موجبة وهي معنوية عند (م=0.10). }
$$

- اما بالنسبة للمتغير (XX) و الذي يمثل صافي الربح قبل الفوائد والضريبة / مجموع الاصول فأن تأثيرة على المتغير التابع غير معنوي.

\section{الاستنتاجات و التوصيات:}

$$
\text { اهم الاستنتاجات التي توصل اليها البحث هي: }
$$

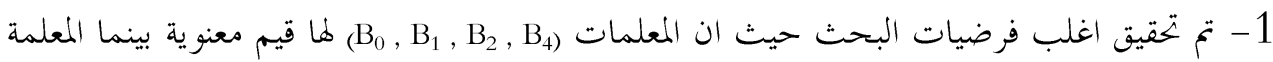

$$
\text { غير معنوية. }
$$

2- ان اعتماد تقنية الانحدار اللوجستي ومن خحلال بناء نموذج احصائي للتنبؤ بوضع الشركة محكن

الاعتماد و التطبيق.

3- تبين ان المتغيرات المستقلة ذات التأثير المعنوي و حسب تسلسل اهميتها هي:

و الذي يمثل المبيعات / بحموع الاصول حيث بلغت قيمة معلمته (B) (B.106).

و الذي يمثل الارباح المحتجزة / بحموع الاصول حيث بلغت قيمة معلمته (B)

و الذي يمثل صافي رأس المال العامل / بحموع الاصول حيث بلغت قيمة معلمته (B (B. 3.394). 4- بلغت نسبة التصنيف الصحيح للنموذج المقترح (84.8\%) و الذي يبين قوة النموذج للتمييز بين

$$
\text { الشر كات الفاشلة و الناجحة. }
$$


5- اثبتت الاختبارات معنوية النموذج الذي تم بناءه بالكامل حيث ان قيمة (X) المحسوبة من نسبة

الامكان الاعظم بلغت (23.974) بلرجة حرية 4 وهي معنوية.

6- ان جودة توفيق النموذج بأختبار Hosmer and Lemeshow) غير معنوي وما يؤكد جودة التوفيق

للنموذ ج

واستناداً الى النتائج التي تم الحصول عليها يمكن ان بنمل اهم التوصيات بالاتي: 1- التوسع بأستخدام تقنية الانحدار اللوجستي بأعتباره أسلوب فاعل لمعرفة اداء الشركات. 2- التأكيد على ديمومة وشفافية نشر كافة البيانات المتعلقة بالشر كات.

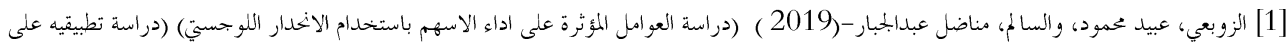

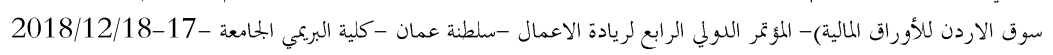

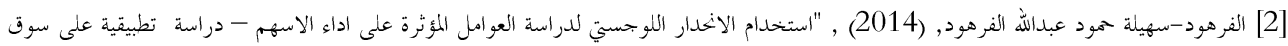

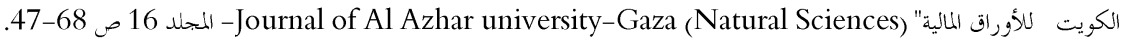
[3] صالخ, عائدة هادي , ( 2015), " تحليل الانحدار اللوجستي لدراسة زمن البقاء لمرضى سرطان الدم " بحلة الادارة والاقتصاد /جامعة كربلاء, المجلد

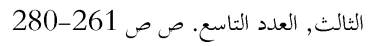

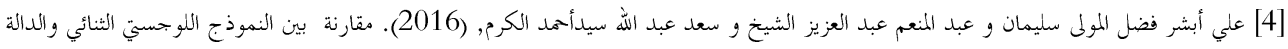

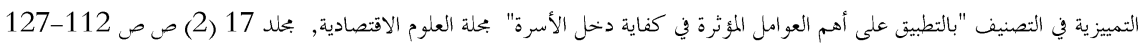

